\title{
Erratum to: Improving the study of proton transfers between amino acid side chains in solution: choosing appropriate DFT functionals and avoiding hidden pitfalls
}

Pedro J. Silva • Marta A. S. Perez • Natércia F. Brás •

Pedro A. Fernandes $\cdot$ M. J. Ramos

Published online: 30 December 2012

(c) Springer-Verlag Berlin Heidelberg 2012

Erratum to: Theor Chem Acc (2012) 131:1179

DOI 10.1007/s00214-012-1179-x

The authors would like to correct an error in the final paragraph of the original publication. The error is an incorrect reference citation to the paper by Nagy and Erhardt. The correct sentence should read as "[...] the prediction of neutral lysine/aspartic acid pairs instead of lysine/aspartate salt bridges [50]". The authors thank Peter Nagy for making them aware of this mistake.

\section{Reference}

50. Nagy PI, Erhardt PW (2010) J Phys Chem B 114:16436-16442

The online version of the original article can be found under doi:10.1007/s00214-012-1179-x.

\section{P. J. Silva}

REQUIMTE/Faculdade de Ciéncias da Saúde, Universidade

Fernando Pessoa, Rua Carlos da Maia, 296,

4200-150 Porto, Portugal

M. A. S. Perez · N. F. Brás · P. A. Fernandes ·

M. J. Ramos $(\square)$

REQUIMTE/Departamento de Química e Bioquímica,

Faculdade de Ciéncias, Universidade de Porto, Rua do Campo

Alegre, s/n, 4169-007 Porto, Portugal

e-mail: mjramos@fc.up.pt 